\title{
Clinical Management of Pediatric Acute-Onset Neuropsychiatric Syndrome: Part I-Psychiatric and Behavioral Interventions
}

\author{
Margo Thienemann, MD, ${ }^{1}$ Tanya Murphy, MD, James Leckman, MD, Richard Shaw, MD, PhD, \\ Kyle Williams, MD, Cynthia Kapphahn, MD, MPH, Jennifer Frankovich, MD, MPH, , Daniel Geller, MD, \\ Gail Bernstein, MD, Kiki Chang, MD, Josephine Elia, MD, and Susan Swedo, MD ${ }^{7}$
}

\begin{abstract}
Objective: This article outlines the consensus guidelines for symptomatic treatment for children with Pediatric Acute-Onset Neuropsychiatric Syndrome (PANS) and Pediatric Autoimmune Neuropsychiatric Syndrome Associated with Streptococcal Infection (PANDAS).

Methods: Extant literature on behavioral, psychotherapeutic, and psychopharmacologic treatments for PANS and PANDAS was reviewed. Members of the PANS Research Consortium pooled their clinical experiences to find agreement on treatment of PANS and PANDAS symptoms.

Results: Current guidelines result from consensus among the Consortium members.

Conclusion: While underlying infectious and inflammatory processes in PANS and PANDAS patients are treated, psychiatric and behavioral symptoms need simultaneous treatment to decrease suffering and improve adherence to therapeutic intervention. Psychological, behavioral, and psychopharmacologic interventions tailored to each child's presentation can provide symptom improvement and improve functioning during both the acute and chronic stages of illness. In general, typical evidence-based interventions are appropriate for the varied symptoms of PANS and PANDAS. Individual differences in expected response to psychotropic medication may require marked reduction of initial treatment dose. Antimicrobials and immunomodulatory therapies may be indicated, as discussed in Parts 2 and 3 of this guideline series.
\end{abstract}

Keywords: consensus guidelines, pediatric acute-onset neuropsychiatric syndrome, pediatric autoimmune neuropsychiatric syndrome associated with streptococcal infection, psychotherapy

\section{Psychiatric and Behavioral Interventions}

$\mathbf{P}$ Ediatric ACute-onset Neuropsychiatric Syndrome (PANS) is defined by the foudroyant (lightning-like) onset of obsessivecompulsive disorder (OCD) or eating restrictions and comorbid symptoms from at least two of seven categories: anxiety (particularly separation anxiety); emotional lability or depression; irritability, aggression, and/or severely oppositional behaviors; deterioration in school performance [related to attention-deficit/ hyperactivity disorder (ADHD)-like behaviors, memory deficits, and cognitive changes]; sensory or motor abnormalities; and somatic signs and symptoms, including sleep disturbances, enuresis, or urinary frequency (Swedo et al. 2012; Chang et al. 2015). Acuteonset cases that are triggered by Group A streptococcal infections may meet diagnostic criteria for both PANS and Pediatric Autoimmune Neuropsychiatric Disorder Associated with Streptococcal

\footnotetext{
${ }^{1}$ Stanford University, Stanford, California.

${ }^{2}$ University of South Florida, St. Petersburg, Florida.

${ }^{3}$ Yale University, New Haven, Connecticut.

${ }^{4}$ Massachusetts General Hospital, Boston, Massachusetts.

${ }^{5}$ Harvard Medical School, Boston, Massachusetts.

${ }^{6}$ University of Minnesota, Minneapolis, Minnesota.

${ }^{7}$ Nemours, Alfred I duPont Hospital for Children, Wilmington, Delaware.

${ }^{8}$ National Institute of Mental Health, Bethesda, Maryland.
}

(c) Margo Thienemann et al. 2017; Published by Mary Ann Liebert, Inc. This article is available under the Creative Commons License CC-BY-NC (http://creativecommons.org/licenses/by-nc/4.0). This license permits non-commercial use, distribution and reproduction in any medium, provided the original work is properly cited. Permission only needs to be obtained for commercial use and can be done via RightsLink. 
infections (PANDAS) (Swedo et al. 1998). As these two syndromes display highly similar symptoms, these guidelines will treat them as a single entity: PANS/PANDAS.

Directly controlling or minimizing symptoms of PANS/PANDAS is essential to decrease suffering, increase the child's ability to receive other important treatments, and overall recovery. As is true in treatment of delirium, the instigating processes (which include infections and inflammatory diseases) and symptoms of PANS/ PANDAS must be addressed simultaneously. Early research in other types of brain injury, including concussion, stroke, and multiple sclerosis, suggest that neural plasticity depends on use and that early rehabilitation has better outcome, supporting the prompt initiation of psychotherapy (Liguez-Lecznar 2013; Prosperini 2015; Turner-Stokes et al. 2015; Ellis 2016). Psychological and behavioral interventions and psychopharmacologic treatment can improve symptoms and decrease impairments during both the acute and chronic stages of illness. Therefore, psychiatric and behavioral interventions should begin as soon as PANS/PANDAS is identified.

\section{Individualizing Treatment}

Since symptom presentations differ, therapies should be tailored to meet each child's specific needs. For example, mildly distressed children may not require pharmacological intervention. In more severe cases, education and support are augmented by symptomspecific behavioral and pharmacologic interventions. The therapeutic interventions may require periodic adjustments, as the PANS/PANDAS symptoms frequently change during the course of illness, such as moving from severe anxiety to aggression or from emotional lability to depression. Antibiotic and immune-based treatments often have dramatic effects, reducing symptoms to a tolerable level or eliminating them completely. In other cases, the treatments fail or a new symptom exacerbation occurs before the child has returned to baseline, resulting in even greater distress and impairment. Given the variability in the course of PANS/PANDAS, clinicians should be cautious about "chasing" the illness by reacting to temporary symptom exacerbations or increasing medication dosages before maximum benefits of the drug and other medical interventions have been realized. The following recommendations are based on the authors' extensive clinical experience and are intended to serve as guidelines for minimizing distress and dysfunction of affected children. Education, supportive and behavioral therapies, and psychoactive medications are the mainstays of symptomatic treatment for PANS/PANDAS. Antimicrobials and immunomodulatory therapies also may be indicated, as discussed in Parts 2 and 3 of this series (Brown et al. 2017; Cooperstock et al. 2017).

\section{Safety}

A comprehensive diagnostic evaluation should be the first step in the treatment of children with PANS/PANDAS, as described in Chang et al. (2015). When taking the child's history, particular attention should be paid to symptoms that pose a risk to the child or his family, such as impulsivity, physical violence or aggression, refusal to eat or drink, and suicidality; if found, they should be immediately addressed with appropriate environmental, educational, and pharmacological measures. Crisis management in PANS/PANDAS is similar to that of other disorders presenting risk of self-harm (AACAP 2012).

Inpatient hospitalization may be required to contain and address dangerous behaviors or if the patient needs closer medical monitoring while starting psychiatric medications (e.g., borderline QTc, or dehydration secondary to restricted oral intake). Ideally, the child would be hospitalized on a pediatric behavioral unit staffed by personnel comfortable with medical procedures and interventions and experienced in the use of behavior modification techniques and containment of aggressive or self-harming behavior. In addition, the unit would allow parents to remain at the child's bedside throughout the stay, since separation anxiety is present in more than $90 \%$ of patients. Such units are rare, and clinicians often must decide between a general pediatrics ward and a pediatric psychiatric unit. While pediatric units allow parents to room-in with the child and offer more expertise in medical procedures and invasive interventions, they are often illequipped to deal with the problematic behaviors of PANS/PANDAS, such as severe agitation, physical combativeness, and compulsive rituals (e.g., refusal to wear clothing). Furthermore, medical units are often overly bright, noisy, and filled with interruptions, which aggravate the sensory sensitivities of children with PANS/PANDAS. In contrast, while child psychiatric units have established protocols for dealing with disruptive or dangerous behaviors, the psychiatric nursing staff may have little experience with invasive procedures and treatments, such as lumbar puncture or administration of intravenous immunoglobulin. In such cases, the child might be transferred to the general pediatrics unit accompanied by staff from the psychiatric unit or, if necessary, to an intensive care unit where the procedures can be performed under sedation. In rare cases, admission to an intensive care unit may be indicated to address life-threatening dehydration and malnutrition due to food refusal (Toufexis et al. 2015).

The majority of children with PANS/PANDAS require some type of school accommodation. Anxieties, OCD symptoms, tics, frequent urination, attention-deficit disorder symptoms, handwriting difficulties, poor cognitive and physical stamina, difficulties with math and processing speed, memory issues, pain, and frequent absences each cause trouble and, in combination, create great challenges to the children and their teachers. Many schools have scarce resources, so persistence, assertiveness, and creativity in the school and family will yield better outcomes. A Student Study Team meeting to arrange for the implementation of either a 504 plan (Section 504 of the Rehabilitation Act) or individualized educational plan (IEP) may be of significant benefit to the child (U.S. Department of Education). The IEP should provide specific individually tailored accommodations that will help the child with PANS/PANDAS attend and benefit from school. Legally, the 504 or IEP must be periodically reviewed, usually annually. PANS children's needs change day to day and month to month. Writing the plans with the most difficult days in mind will assure that the child can receive adequate accommodations without revisiting the 504 or IEP process often. Typical accommodations during flares of illness might include the following:

General—Excusing the child's absences and not requiring makeup assignments or tests.

Separation anxiety-allowing a parent to be in or near the classroom (perhaps helping out).

OCD symptoms - excusing the child from certain activities, allowing him to complete assignments using alternate methods (e.g., typing homework, rather than erasing and rewriting repeatedly; listening to audio books, rather than reading and rereading).

Urinary urgency/frequency-leaving the class without asking permission.

Dysgraphia or handwriting difficulties-having a note-taker in class, dictating tests and homework, enlarging worksheets, writing on large grid paper, and using a keyboard, voicerecognition software, or audio recorder.

Dyscalculia and math difficulties-using a calculator or times table and working with a resource teacher or tutor. 
Slowed processing speed-decreasing the number and length of assignments, allowing extra time for tests and in-class assignments, and giving directions in written and oral form, and Poor physical and cognitive stamina, pain-shorter school day with reduced academic load, less homework, rest periods during the day (perhaps in the nurse's office), and omitting or adapting physical education requirements.

When acute exacerbations of PANS/PANDAS symptoms have abated, these accommodations should be re-evaluated. Attending school and doing school work may be part of an appropriate psychotherapeutic plan. As the child's function improves, the accommodations can be peeled back as symptoms improve and to align with therapeutic goals.

\section{Family Support and Education}

The emotional and behavioral symptoms of PANS/PANDAS can stress patients and their families. Often one parent must stay home from work to provide round-the-clock care for the child with PANS/ PANDAS. In addition to providing comfort and care, the parents must also find and coordinate medical care and interact with schools to secure necessary accommodations (and care for siblings).

Clinicians should ensure that families understand the clinical course of PANS/PANDAS, including its unpredictably episodic pattern. In severe cases, it is not uncommon for a child to insist on obsessive compulsive rituals, to resist any separation from his parent at all, or to become enraged at without apparent provocation. At such times, interventions should be aimed at keeping the child safe until the anxiety or rage subsides. Minimizing transitions and numbers of activities, providing rest times, decreasing noxious sensory stimuli, and removing objects that might hurt the child or others may help to decrease the number and severity of "meltdowns." Once the episode has subsided, parents should be encouraged to resume their normally effective parenting strategies, including setting necessary limits, reinforcing desirable behaviors, and ignoring or punishing unwanted behaviors by removing privileges. Often, the affected child can help parents to distinguish undesirable behaviors requiring intervention from those that are an unfortunate manifestation of his PANS/ PANDAS symptomatology (Motluck 2013).

Parents, themselves, suffer with the burden of caring for their PANS/PANDAS afflicted children. Many report feeling traumatized by the sometimes lengthy course of seeking medical care and from seeing their child ill; they often live fearing relapse. National and regional support networks have formed, which may be helpful to address this trauma and skills groups for PANS/PANDAS, parents are also being piloted.

\section{Behavioral and Pharmacological Interventions for PANS/PANDAS}

Cognitive behavior therapy (CBT) (specifically exposure/response prevention [ERP]) and minimizing family accommodation to OCD behaviors have been repeatedly shown to be among the most effective interventions for pediatric OCD (OCD 2004, Lebowitz et al. 2011). While large-scale studies of these interventions in PANS/PANDAS have not been conducted, pilot studies suggest behavioral therapies are still highly effective in treating the OCD symptoms of children with PANS/PANDAS (Storch et al. 2006). Treatment with CBT/ERP should be initiated as soon as possible to maximize benefits. During the acute phase of illness, the child may not be ready to fully participate in CBT because of overwhelming OCD or anxiety, sensory sensitivities, emotional lability, and/or cognitive impairments. However, it is helpful when parents learn strategies to "hold the line" and prevent symptoms from further worsening through their inadvertent accommodation to the child's irrational fears (March et al. 1998; Lebowitz et al. 2011, 2014; Motluck 2013; Sukhodolsky et al. 2013). Understanding and applying CBT and ERP strategies will decrease distress and interference from symptoms in the short term and can provide tools that can be used life-long to manage OCD, anxiety, and life stressors.

Psychoactive medications can also be quite helpful in the management of PANS/PANDAS symptoms. Although controlled trials have not been done, clinical experience suggests that anxiety, OCD, and other symptoms of PANS/PANDAS respond to the same medications used to treat the symptoms in non-PANS/PANDAS cases. However, the PANS/PANDAS clinical presentations are often more complex, with multiple symptom targets presenting simultaneously. Furthermore, the episodic symptom course can make it difficult to discern responses to pharmacologic interventions that require days or weeks of administration before benefits are realized from the independent relapses and remittances of PANS/PANDAS symptoms. Some experienced clinicians report that PANS/PANDAS patients appear to be more sensitive to adverse effects of psychotropic medications, including agitation, dystonia, or catatonia, but no controlled studies speak to these anecdotal reports. Thus, clinicians are advised to "start low and go slow" in prescribing these medications-beginning with dosages $1 / 4$ (or less) of typical and using an extended upward titer. Benzodiazepines may be a good first treatment for PANS/PANDAS, as they may address the child's anxiety, agitation, aggression, insomnia, and other symptoms. Clinicians and parents should be aware that pediatric patients may experience severe disinhibition when treated with benzodiazepines, although the medications are otherwise well tolerated in youth with PANS/PANDAS.

\section{Management of Specific PANS/PANDAS Symptoms}

\section{Obsessive compulsive (OCD) symptoms}

In general, management of obsessions and compulsions follows the usual standard of care for pediatric OCD (Geller and March 2012). Evidence-based therapies include CBT, parent management training, and medications. A small study of intensive CBT with family involvement found that OCD symptoms improved in PANDAS patients during the current episode and benefits carried over to subsequent flares (Storch et al. 2006). Applying parent management techniques (PMT), which include encouraging families to not participate in behaviors accommodating the child's avoidance and rituals, positively reinforcing desired behaviors, setting clear limits, expectations, and consequences, and establishing reward systems, will likely improve symptoms and child compliance. PMT are particularly useful when PANS patients are not ready or willing to use CBT themselves (Lebowitz et al. 2014).

Selective serotonin reuptake inhibitors (SSRI) are the preferred medication for treatment of OCD in PANS, based on multiple placebo-controlled clinical trials (Geller 2003; Grados and Riddle 2001; Pediatric OCD Treatment Study Team 2004, 2011) (Gabbay and Coffey 2003; Coffey and Wieland 2007). Fluoxetine, sertraline, fluvoxamine, and clomipramine are Food and Drug Administration (FDA) approved for treating pediatric OCD and have the most evidence to support their use (ibid). Clinical experience suggests both that SSRI's may be helpful and that using a low dose and slow titration minimizes the risk of side effects. Upward titrations should be adjusted no faster than 2-week intervals. Frequent monitoring is recommended to ensure that side effects are not becoming troublesome. In one, 14 of 38 children with PANDAS 
experienced behavioral activation when treated with an SSRI; symptoms included hyperactivity, mania, disinhibited behavior, worsening OCD behavior, aggression, irritability, agitation, and suicidality (Murphy et al. 2006). In one boy with PANS, when $5 \mathrm{mg} /$ day of fluoxetine was discontinued, suicidal behaviors ceased and he was able to tolerate $2 \mathrm{mg}$ /day of fluoxetine when the medication was reintroduced (Murphy et al. 2006). SSRI side effects can match symptoms one is trying to relieve, so careful attention to the relationship of medication dose and timing of symptom change, along with monitoring the course of underlying PANS itself, is necessary to optimize SSRI treatment.

Antipsychotic medications, such as risperidone $(0.125-1 \mathrm{mg})$ or aripiprazole $(0.5-2.0 \mathrm{mg})$, should be reserved for incapacitating OCD. An electrocardiogram (EKG) should be obtained before and during treatment with antipsychotics to monitor the QTc intervalexcessive prolongation (QTc $>450$ mseconds) is a contraindication to use of antipsychotic medications.

\section{Restriction of food or fluid intake}

The sudden onset of reduced and restricted food intake fulfills the first criterion for PANS, even in the absence of more typical OCD symptoms (Swedo et al. 2012; Toufexis et al. 2015). Medical evaluation should be done to rule out other medical disorders (e.g., eosinophilic esophagitis, structural or neurological causes of dysphagia, and nausea) and assess for medical instability related to restricted intake of food and/or fluids. For those with severely limited oral intake, assessment should include checking orthostatic vital signs, EKG, and electrolytes, including phosphorus and magnesium and monitoring for refeeding syndrome. Hospitalization may be required for children who are medically unstable because of restricted intake of food and/or fluids (Chang et al. 2015; Golden et al. 2015).

In treating children with intake disturbances related to PANS, the focus should be on maintaining adequate nutrition and hydration while treating underlying brain inflammation. Feeding tubes may be necessary, at least during the acute phases of the illness. The severity and duration of the eating disturbances can vary. For example, in four reported cases of infection-triggered anorexia nervosa, treatment with antibiotics improved symptoms remarkably (Sokol 2000). However, many PANS patients experience ongoing eating abnormalities and require interventions used in other eating disorder treatments to directly target other factors contributing to the eating restrictions (Lock 2015).

For patients with high anxiety and compulsive behaviors, an exposure and response prevention approach may prove helpful. This involves gradual exposure to foods or situations that cause anxiety or fear, within a support framework that encourages incremental progress toward expanding diet and increasing intake. Occupational therapy interventions that include targeting posture, breathing, and relaxation may be helpful adjuncts, especially for patients who fear of choking or vomiting. When the problems with eating involve obsessions or compulsions, a psychopharmacological approach similar to that used for OCD may help. However, in cases of significant malnutrition, medication may not help until the child has restored weight, and malnourished patients may be at increased risk of adverse side effects.

\section{Tics}

Based on preliminary studies, as many as $70 \%$ of PANS/ PANDAS patients either present with or develop tics (Murphy et al. 2015; Swedo et al. 2015). PANS patients with tics have been re- ported to be more severely affected at home and at school, with poorer school performance, handwriting, visual-spatial memory, and self-esteem (Murphy et al. 2015). Some PANS/PANDAS patients' tics first present with complex motor and vocal tics, rather than presenting with a progression from simple tics to complex motor and phonic tics that is typical of Tourette syndrome (Tucker et al. 1996).

In most cases, tics are not considered a treatment target unless they cause pain, significant interference in function, or create unresolvable embarrassment or teasing. Comprehensive behavioral intervention for tics (CBIT), habit reversal training (HRT), and cautious monitored pharmacotherapy are the treatments of choice (Piacentini et al. 2010; Murphy et al. 2013). In HRT, children learn to be aware of their tics and their antecedent premonitory urge. They help design a "competing response," which is a motor behavior that makes doing the tic impossible. When children practice this, resisting or suppressing the tic, as in response prevention for $\mathrm{OCD}$, they stop reinforcing the urge and the urge frequency diminishes. Examples of competing responses include staring or looking up to extinguish a blinking tic and slow, rhythmic diaphragmatic breathing for vocal tics. Relaxation training, analyzing, and addressing situations that worsen tics and family reinforcement of applying HRT strategies comprise other elements of CBITC. Exposure with Response Prevention may also be effective intervention for severe tics (Wile and Pringsheim 2013).

Many clinicians consider the alpha-2 adrenergic agonists, clonidine, and guanfacine to be the first-line pharmacologic intervention for tics. However, a recent meta-analysis has suggested that alpha-2 agonists may have minimal benefit in tic patients without ADHD (Weisman et al. 2013). Antipsychotics may be indicated for severe tics, although their use should be restricted for as short a duration as necessary. Haloperidol, pimozide, and aripiprazole have FDA approval for the treatment of Tourette Syndrome. Of the atypical antipsychotics, risperidone also has evidence supporting its use for the treatment of tics, but does not have an FDA indication (Budman 2014). In addition, a recent meta-analysis of the available scientific literature found no significant difference in the efficacy of four antipsychotic agents evaluated (risperidone, pimozide, haloperidol, and ziprasidone) (Weisman et al. 2013). As described above, an EKG should be obtained before, and during, treatment with antipsychotic medications to rule out a prolonged QTc.

\section{Irritability and aggression}

Irritability, aggression, and unprovoked violent behaviors are among the most troubling symptoms of PANS. Parents frequently report mood lability and impulsive aggression in children with PANS/PANDS. Sensory sensitivities, irritability, fatigue, anxiety, and cognitive changes may cause the child to adapt poorly to environmental changes, making them vulnerable to aggressive behaviors. The episodes may last for 45-60 minutes or longer, but within a few minutes of the rage subsiding, the child is "back" and expresses remorse for his tantrum, often not knowing or remembering why they behaved as they did.

Environmental interventions may decrease the frequency of aggressive behavior. Modulating stimulation, minimizing demands, and promoting adequate nutrition and sleep may each help. In the moment, distraction may be one of the most effective tools, for example, the use of favorite toys (that are unlikely to be used as weapons), singing, dancing, television, food, crying, and acting silly may re-engage the child's "cortex" and truncate the episode. Designing a home plan for calling for help and remaining 
nonpunitive with the child is important. Although calling 911 may seem like an extreme solution to a child's behavior, it is sometimes necessary in order for the parents to protect themselves and their children from harm, to reassure children that their behavior can be contained, and to not undermine established behavior plans. If rages are frequent, it may be worthwhile to educate local police officers about the nature of the illness, as, understandably, police might misinterpret rageful episodes as acting-out behavior.

For irritability related to anxiety, benzodiazepines may be the safest and most effective psychotropic with which to begin treatment. Antipsychotic medications or mood stabilizers have been shown to reduce the frequency and intensity of aggressive behaviors. Despite their associated adverse effects, they may be indicated for children whose aggressive behavior risks harming themselves or others. The medications may be prescribed on an as-needed basis (if possible to administer then) or on a regular schedule. Risperidone and aripiprazole are FDA approved for treating irritability in autistic children.

For patients with aggression secondary to encephalitis, helpful interventions have included the following:

- Diphenhydramine in doses from 12.5 to $50 \mathrm{mg}$. Side effects include paradoxical excitation or confusion. May be given orally, intramuscularly (IM), or IV.

- Benzodiazepines: Lorazepam at doses beginning at $0.5 \mathrm{mg}$. Also can cause agitation and disinhibition. May be given orally, IM, or IV.

- Antipsychotics: Risperidone, aripiprazole, or haloperidol may be helpful for children with aggression and rage. Haloperidol is started at $0.025 \mathrm{mg} / \mathrm{kg} /$ day in divided doses going up to a maximum of $0.05 \mathrm{mg} / \mathrm{kg} / \mathrm{day}$ in divided doses (compared with the typical starting dose of $0.5 \mathrm{mg}$ for larger children and adolescents). May be given orally, IM, or IV. When given orally or IM, it should be given with diphenhydramine. Due to the risk of drug effects and drug-drug interactions, an EKG must be checked for QTc prolongation when using antipsychotics.

\section{Anxiety}

Separation anxiety frequently interferes with PANS children's comfort and family function, restricting both the child's and parent's activity. Fortunately, with treatment of the underlying causes of PANS, the suffering and burden may be short-lived. If symptoms are resolving, accommodating the anxiety for a short time may be tolerable. As soon as possible, the child should go back to his bedroom and classroom. When separation anxiety persists, the parent may become exhausted and require respite, so efforts should be made to integrate other attachment figures into care. Parents may also benefit from behavioral interventions for caregivers, if family accommodation is part of the clinical picture (Jones et al. 2015; Norman et al. 2015).

CBT is helpful for both generalized and separation anxiety and may involve the cognitive skills of reappraisal and problem solving. Children learn to assess the probability and the likely severity of feared events and generate backup plans should the problem arise. For example, a child might work through the probability that no one would pick him up from school, articulate realistic outcomes of being left at school, and list possible actions the child might take if left. The child might role-play those actions and, with parents, and design a gradual exposure plan with rewards for following through. Notifying the school of the issue and identifying "safe" individuals and ways to access them may aid in this endeavor.
Should pharmacotherapy be necessary, benzodiazepines may be used temporarily. Antihistamines, gabapentin, or clonidine may help; dosages for these have not been empirically established, so the recommendation is to begin with small doses and titrate to effects and side effects. Administration of SSRI's should follow guidelines outlined in the section above on OCD.

\section{ADHD symptoms}

Some ADHD symptoms seen in PANS/PANDAS may be manifestations of underlying ADHD. PANS/PANDAS children's hyperactivity may reflect anxiety, complex tic sequences, restlessness exacerbated by cognitive and physical fatigue, sensory overload, pain or urge to urinate, or agitated catatonia (Elia et al. 2005). Inattention may reflect sleep deprivation, mood lability, intrusive thoughts of OCD, or the cognitive "fog" some PANS/ PANDAS patients describe. Academic frustrations may contribute to impulsivity and disruptive behaviors.

If the treatment team considers symptoms consistent with ADHD, management of ADHD symptoms in the context of PANS/ PANDAS is based on our understanding of ADHD management in general. Typical classroom accommodations, possibly with pharmacotherapy, may help the child tolerate the classroom and help the classroom tolerate the child. Useful changes have included preferential seating (front and side), permission to take breaks without interrupting the class and without interruption (e.g., to do sham errands for the teacher), extended time for tests, and resource assistance for math and executive function support and training.

Stimulants (methylphenidate and amphetamine compounds), the first-line medication for idiopathic ADHD, may be tolerated and helpful. Methylphenidate may be preferable to amphetamine preparations as they may have a smaller risk of exacerbating tics or compulsive behaviors in children with non-PANS/PANDAS ADHD (Borcherding 1990; Castellanos 1997; Pringsheim 2011). Some have suggested that amphetamine formulations pose a higher risk for neurotoxicity, so methylphenidate preparations are preferable (Berman et al. 2009). Atomoxetine, a second line treatment for ADHD symptoms, appears to possess anti-inflammatory properties through modulation of noradrenaline signaling, and in so reduces the expression of proinflammatory cytokines, downregulates cell adhesion molecules important in leukocyte infiltration, and strengthens the blood-brain barrier (O'Sullivan et al. 2010). Alpha-2 adrenergic agonists, guanfacine, and clonidine are less helpful for ADHD symptoms, but may be the "treatment of choice" for PANS/PANDAS hyperactivity and impulsivity because they may also produce improvements in tics, anxiety, and sleep disruptions.

\section{Sleep disturbances}

Disordered sleep in PANS/PANDAS may be a function of separation anxiety, OCD bedtime rituals, enuresis, nightmares, temperature dysregulation, sleep apnea (sometimes with large tonsils), or rapid eye movement (REM) Sleep Behavioral Disorder. In the latter condition, muscle tone is maintained during REM sleep, permitting movement during dreams. REM Sleep Behavioral Disorder, associated with neuroinflammation in other disorders, has been observed in 12 of 15 children with PANS/PANDAS studied at NIMH (Gaughan et al. 2016). REM Sleep Behavioral Disorder also may occur as an adverse effect of SSRI treatment (Lloyd et al. 2012). 
Employing good sleep hygiene improves the likelihood of sleeping well, even for children with PANS/PANDAS. The principles of sleep hygiene include the following:

- Scheduling regular times to go to bed and get up (even on weekends) and to set circadian and sleep-wake rhythms

- Healthy sleep habits (e.g., having a consistent, short, pleasant bedtime routine)

- Comfortable sleep environment (e.g., cool, dark, and quiet),

- Avoid caffeine in the evening and "wind down" toward bedtime, restricting exercise, high-energy play, and heavy meals (Moore 2010)

- Restrict evening use of electronic games and toys and turn off televisions for at least an hour before bedtime, as the blue light of electronic screens can delay sleep onset and shorten sleep duration (Hale and Guan 2015)

If behavioral interventions fail to improve sleep, diphenhydramine, melatonin, cyproheptadine, clonidine, trazodone, or zolpidem may be useful; each has anecdotal report of benefit in PANS. However, controlled studies are lacking, and none of these medications is approved by the FDA for use in children. A low dose may be adequate and desirable to minimize side effects.

\section{Depression}

Dysphoria and depressive symptoms are common in PANS/ PANDAS, particularly during later stages of the illness (Frankovich et al. 2015; Murphy et al. 2015; Swedo et al. 2015). For mild mood symptoms, supportive interventions, such as individual or family therapy, may be sufficient. However, children with fullblown depression, suicidal ideation, or self-injurious behavior require more intensive treatment. Psychotherapy or psychotherapy combined with an SSRI is the standard of care for youth with major depressive episodes (Hopkins et al. 2015). Elements of evidenced psychotherapies for depression seem particularly useful for children with PANS. In Interpersonal Therapy for Adolescents, the therapist, family, and school recognize that the child is "sick" and unable to function at usual capacity (Mufson et al. 2004). In CBT for depression, planning pleasant (and for PANS patients, probably low-key) experiences comprises part of the behavioral element.

When depressive symptoms are sustained and/or impairing, clinical experience suggests treatment with an SSRI or bupropion may be helpful. The medications are started at a low dose, and parents are advised to watch for emergent adverse effects, particularly worsening mood, irritability, agitation, hyperactivity, suicidal thoughts, or sleeplessness. Likewise, when a child's symptoms are consistent with bipolar mood disorder, medications for this indication, given with the same caution, could provide benefit. In families with a history of bipolar disorder, antidepressant medications must be used with extreme caution because of the increased risk of precipitating mania.

\section{Psychosis}

At least $25 \%$ of children with PANS/PANDAS report auditory, olfactory, or visual hallucinations (distinct from OCD intrusive images, Frankovich et al. 2015) If disturbing or impairing, then antipsychotic medications may be warranted, despite their associated adverse effects. These are used typically for a short duration to manage acute psychosis and agitation. Medication choice should consider minimizing adverse effects and being aware of interactions with other medications. For chronic psychotic symptoms, diagnosis and ongoing treatment need to be reassessed. In other encephalitides, the following have been reported helpful: (Kuppuswamy et al. 2014)

- Aripiprazole (lupus, anti-NMDAR)

- Risperidone (Wilson's, Hashimoto's, anti-NMDAR)

- Olanzapine (anti-NMDAR)

- Haloperidol (anti-NMDAR)

- Quetiapine

\section{Pain}

While not often volunteered as a complaint, upon questioning, it has been found that pain is a common comorbidity in PANS patients (Frankovich et al. 2015). Musculoskeletal pain in PANS is broadly caused by two factors: inflammatory (arthritis) or a pain amplification syndrome due to pain processing abnormalities. Pain due to arthritis typically precedes or presents simultaneously with the PANS symptoms. Wide-spread pain and other pain amplifications syndromes more commonly present during the recovery phase of PANS. If the pain disorder is not diagnosed and treated early, it can develop into a refractory pain syndrome. When widespread pain is present, patients often report other forms of sensory amplification (increased sensitivity to light, sound, and sometimes smells), abdominal pain, headaches, muscle aches, waking unrefreshed, daytime fatigue, "foggy brain," and depression. Physical examination may reveal areas of tenderness to palpation in the classic distribution for fibromyalgia. Patients who report both pain and stiffness upon awakening or after prolonged stationary positions should be assessed further for arthritis, enthesitis, and inflammatory back pain. Involvement of a pediatric rheumatologist and/or pain specialist, occupational therapist, and physical therapist is necessary for treating these pain conditions. Alleviation of pain will secondarily improve emotional and physical functioning.

\section{Conclusion}

The treatment of PANS/PANDAS is symptom based and closely mirrors that of childhood mental illness of idiopathic etiologies. Mainstays of treatment include psychoeducational, psychotherapeutic, behavioral, family, school-based, and pharmacological interventions, which reduce suffering and improve functioning until the immunologic and infectious processes are addressed. Fortunately, many patients with PANS/PANDAS will recover completely and symptomatic treatments can be discontinued. Others are less fortunate and have ongoing symptoms that require continuous intervention and accommodation. At present, we are unable to predict the individual differences in the course and prognosis of individual PANS/PANDAS cases. Additional research is needed to understand the clinical heterogeneity associated with these cases.

\section{Clinical Significance}

Until further research informs clinicians about effective treatments for behavioral and psychiatric interventions for children with PANS and Pediatric Autoimmune Neuropsychiatric Syndrome Associated with Streptococcal Infection (PANDAS), current literature and clinical experience must guide clinicians treating these children. This article fills the gap between empiric therapy and current knowledge by conveying consensus guidelines for symptomatic treatment of children with PANS and PANDAS.

\section{Disclosures}

T.M. reports grants from Auspex Pharmaceuticals grants from National Institute of Mental Health, Shire Pharmaceuticals, Pfizer, 
Inc., F. Hoffmann-La Roche Ltd., AstraZeneca Pharmaceuticals, Centers for Disease Control, Massachusetts General Hospital, Sunovion Pharmaceuticals, Neurocrine Biosciences, Psyadon Pharmaceuticals, and PANDAS Network, and personal fees from International OCD Foundation, Tourette Syndrome Association. J.L. "I do not consider that I have any conflicts of interest with regard to the guidelines. That said, here is what JAACAP requires that I report: J.F.L. has received grant or research support from the National Institutes of Health, the UBS Optimus Foundation, and the Open Road Alliance. He has served on the advisory boards of the Brain and Behavior Research Foundation, Fondazione Child, the European Multicentre Tics in Children Studies, and How I Decide. He has authored the Yale Global Tic Severity Scale assessment tool, which is open access. He has received honoraria from the European Society for the Study of Tourette Syndrome and the Brazilian Psychiatric Association. He has received royalties from John Wiley and Sons, McGraw Hill, and Oxford University Press. He has received travel expenses from the University of Illinois-Chicago, Cornell Weill Medical College, the Medical University of South Carolina, Rutgers University, the British Academy, and the Brazilian Psychiatric Association. He has received additional support from Anne Çocuk Eğitim Vakfi (AÇEV; Mother Child Education Foundation) and private donors." K.C. is an unpaid consultant for GSK, Lilly, and BMS. He is on the DSMB for Sunovion. In the past 3 years, he has received research support from GSK and Merck and has been a consultant for Actavis and Janssen. Remaining authors do not have any conflict of interest.

\section{References}

Berman SM, Kuczenski R, McCracken JT, London ED: Potential adverse effects of amphetamine treatment on brain and behavior: A review. Mol Psychiatry 14:123-142, 2009.

Borcherding BG, Keysor CS, Rapoport JL, Elia J, Amass J: Motor/ vocal tics and compulsive behaviors on stimulant drugs: Is there a common vulnerability? Psychiatry Res 33:83-94, 1990.

Brown K, Farmer C, Farhadian B, Hernandez J, Thienemann M, Frankovich J: Pediatric acute-onset neuropsychiatric syndrome (PANS)-response to oral corticosteroid burts: An observational study. J Child Adolesc Psychopharmacol 2017 [Epub ahead of print]; DOI: 10.1089/cap.2016.0139.

Budman CL: The role of atypical antipsychotics for treatment of Tourette's syndrome: An overview. Drugs 74:1177-1193, 2014.

Castellanos FX, Giedd JN, Elia J, Marsh WL, Ritchie GF, Hamburger SD, Rapoport JL: Controlled stimulant treatment of ADHD and comorbid Tourette's syndrome: Effects of stimulant and dose. J Am Acad Child Adolesc Psychiatry 36:589-596, 1997.

Chang K, Frankovich J, Cooperstock M, Cunningham MW, Latimer ME, Murphy TK, Pasternack M, Thienemann M, Williams K, Walter J, Swedo SE: Clinical evaluation of youth with pediatric acute-onset neuropsychiatric syndrome (PANS): Recommendations from the 2013 PANS Consensus Conference. J Child Adolesc Psychopharmacol 25:3-13, 2015.

Coffey B, Wieland N: Tics, anxiety, and possible PANDAS in an adolescent. J Child Adolesc Psychopharmacol 17:533-538, 2007.

Cognitive-behavior therapy, sertraline, and their combination for children and adolescents with obsessive-compulsive disorder: The Pediatric OCD Treatment Study (POTS) randomized controlled trial. JAMA 292:1969-1976, 2004.

Cooperstock MS, Swedo SE, Pasternack MS, Murphy TK: Clinical management of pediatric acute-onset neuropsychiatric syndrome: Part III-Treatment and prevention of infections. J Child Adolesc
Psychopharmacol, 2017 [E-pub ahead of print]; DOI: 10.1089/ cap.2016.0151.

Elia J, Dell ML, Friedman DF, Zimmerman RA, Balamuth N, Ahmed AA, Pati S: PANDAS with catatonia: A case report. Therapeutic response to lorazepam and plasmapheresis. J Am Acad Child Adolesc Psychiatry 44:1145-1150, 2005.

Ellis MJ, Leddy J, Willer B: Multi-disciplinary management of athletes with post-concussion syndrome: An evolving pathophysiological approach. Front Neurol 7:136, 2016.

Frankovich J, Thienemann M, Pearlstein J, Crable A, Brown K, Chang $\mathrm{K}$ : Multidisciplinary clinic dedicated to treating youth with pediatric acute-onset neuropsychiatric syndrome: Presenting characteristics of the first 47 consecutive patients. J Child Adolesc Psychopharmacol 25:38-47, 2015.

Gabbay V, Coffey B: Obsessive-compulsive disorder, tourette's disorder, or pediatric autoimmune neuropsychiatric disorders associated with Streptococcus in an adolescent? Diagnostic and therapeutic challenges. J Child Adolesc Psychopharmacol 13:209-212, 2003.

Gaughan T, Buckley A, Hommer R, Grant P, Williams K, Leckman JF, Swedo SE: Rapid eye movement sleep abnormalities in child with Pediatric Acute-Onset Neuropsychiatric Syndrome (PANS). J Clin Sleep Med 12:1027-1032, 2016.

Geller DA, Biederman J, Stewart SE, Mullin B, Martin A, Spencer T, Faraone SV: Which SSRI? A meta-analysis of pharmacotherapy trials in pediatric obsessive-compulsive disorder. Am J Psychiatry 160:1919-1928, 2003.

Geller DA, March J: Practice parameter for the assessment and treatment of children and adolescents with obsessive-compulsive disorder. J Am Acad Child Adolesc Psychiatry 51:98-113, 2012.

Golden NH, Katzman DK, Sawyer SM, Ornstein RM, Rome ES, Garber AK, Kohn M, Kreipe RE: Update on the medical management of eating disorders in adolescents. J Adolesc Health 56:370-375, 2015.

Grados MA, Riddle MA: Pharmacological treatment of childhood obsessive-compulsive disorder: From theory to practice. J Clin Child Psychol 30:67-79, 2001.

Hale L, Guan S: Screen time and sleep among school-aged children and adolescents: A systematic literature review. Sleep Med Rev 21:50-58, 2015.

Hopkins K, Crosland P, Elliott N, Bewley S: Diagnosis and management of depression in children and young people: Summary of updated NICE guidance. BMJ 350:h824, 2015.

Jones JD, Lebowitz ER, Marin CE, Stark KD: Family accommodation mediates the association between anxiety symptoms in mothers and children. J Child Adolesc Mental Health 27:41-51, 2015.

Kuppuswamy PS, Takala CR, Sola CL: Management of psychiatric symptoms in anti-NMDAR encephalitis: A case series, literature review and future directions. Gen Hospital Psychiatry 36:388-391, 2014.

Lebowitz ER, Omer H, Hermes H, Scahill L: Parent training for childhood anxiety disorders: The SPACE Program. Cogn Behav Pract 21:456-469, 2014.

Lebowitz ER, Vitulano LA, Mataix-Cols D, Leckman JF: Editorial perspective: When OCD takes over...the family! Coercive and disruptive behaviours in paediatric obsessive compulsive disorder. J Child Psychol Psychiatry 52:1249-1250, 2011.

Lloyd R, Tippmann-Peikert M, Slocumb N, Kotagal S: Characteristics of REM sleep behavior disorder in childhood. J Clin Sleep Med 8:127-131, 2012.

Lock J: An update on evidence-based psychosocial treatments for eating disorders in children and adolescents. J Clin Child Adolesc Psychol 44:707-721, 2015.

March JS, Mulle K: OCD in Children \& Adolescents: A CognitiveBehavioral Treatment Manual. New York, Guilford Press, 1998.

Moore M: Bedtime problems and night wakings: Treatment of behavioral insomnia of childhood. J Clin Psychology 66:1195-1204, 2010. 
Motluck A: A feverish debate: Are common bacterial infections making children mentally ill? Walrus 6:38-47, 2013.

Mufson L, Dorta K, Wickramaratne P, Nomura Y, Olfson M, Weissman MM: A randomized effectiveness trial of interpersonal psychotherapy fordepressed adolescents. Arch Gen Psychiatry 61: 577-584, 2004.

Murphy TK, Lewin AB, Storch EA, Stock S: Practice parameter for the assessment and treatment of children and adolescents with tic disorders. J Am Acad Child Adolesc Psychiatry 52:1341-1359, 2013.

Murphy TK, Parker-Athill EC, Lewin AB, Storch EA, Mutch PJ: Cefdinir for recent-onset pediatric neuropsychiatric disorders: A pilot randomized trial. J Child Adolesc Psychopharmacol 25:57-64, 2015.

Murphy TK, Storch EA, Strawser MS: Selective serotonin reuptake inhibitor-induced behavioral activation in the PANDAS subtype. Primary Psychiatry 13:87, 2006.

Norman KR, Silverman WK, Lebowitz ER: Family accommodation of child and adolescent anxiety: Mechanisms, assessment, and treatment. J Child Adolesc Psychiatr Nurs 28:131-140, 2015.

O'Sullivan JB, Ryan KM, Harkin A, Connor TJ: Noradrenaline reuptake inhibitors inhibit expression of chemokines IP-10 and RANTES and cell adhesion molecules VCAM- 1 and ICAM-1 in the CNS following a systemic inflammatory challenge. J Neuroimmunol 220:34-42, 2010.

Piacentini J, Woods DW, Scahill L, Wilhelm S, Peterson AL, Chang S, Ginsburg GS, Deckersbach T, Dziura J, Levi-Pearl S, Walkup JT: Behavior therapy for children with Tourette disorder: A randomized controlled trial. JAMA 303:1929-1937, 2010.

Pringsheim T, Steeves T: Pharmacological treatment for attention deficit hyperactivity disorder (ADHD) in children with comorbid tic disorders. Cochrane Database Syst Rev 13:CD007990, 2011.

Prosperini L, et al. Functional and structural brain plasticity enhanced by motor and cognitive rehabilitation in multiple sclerosis. Neural Plasticity 481574, 2015.

Sokol MS: Infection-triggered anorexia nervosa in children: Clinical description of four cases. J Child Adolesc Psychopharmacol 10: 133-145, 2000.

Storch EA, Murphy TK, Geffken GR, Mann G, Adkins J, Merlo LJ, Duke D, Munson M, Swaine Z, Goodman WK: Cognitivebehavioral therapy for PANDAS-related obsessive-compulsive disorder: Findings from a preliminary waitlist controlled open trial. J Am Acad Child Adolesc Psychiatry 45:1171-1178, 2006.

Sukhodolsky DG, Gorman BS, Scahill L, Findley D, McGuire J: Exposure and response prevention with or without parent management training for children with obsessive-compulsive disorder complicated by disruptive behavior: A multiple-baseline acrossresponses design study. J Anxiety Disord 27:298-305, 2013.

Swedo SE, Leckman JF, Rose NR: From research subgroup to clinical syndrome: Modifying the PANDAS criteria to describe PANS (Pediatric Acute-onset Neuropsychiatric Syndrome). Pediatr Therapeut 2:113, 2012.

Swedo SE, Leonard HL, Garvey M, Mittleman B, Allen AJ, Perlmutter S, Lougee L, Dow S, Zamkoff J, Dubbert BK: Pediatric autoimmune neuropsychiatric disorders associated with streptococcal infections: Clinical description of the first 50 cases. Am J Psychiatry 155:264-271, 1998.

Swedo SE, Seidlitz J, Kovacevic M, Latimer ME, Hommer R, Lougee L, Grant P: Clinical presentation of pediatric autoimmune neuropsychiatric disorders associated with streptococcal infections in research and community settings. J Child Adolesc Psychopharmacol 25:26-30, 2015.

Toufexis MD, Hommer R, Gerardi DM, Grant P, Rothschild L, D'Souza P, Williams K, Leckman J, Swedo SE, Murphy TK: Disordered eating and food restrictions in children with PANDAS/ PANS. J Child Adolesc Psychopharmacol 25:48-56, 2015.

Tucker DM, Leckman JF, Scahill L, Wilf GE, LaCamera R, Cardona L, Cohen P, Heidmann S, Goldstein J, Judge J, Snyder E, Bult A, Peterson BS, King R, Lombroso P: A putative poststreptococcal case of OCD with chronic tic disorder, not otherwise specified. J Am Acad Child Adolesc Psychiatry 35:1684-1691, 1996.

Turner-Stokes L, Pick A, Nair A, Disler PB, Wade DT: Multidisciplinary rehabilitation for acquired brain injury in adults of working age. Cochrane Database Syst Rev 12:CD004170, 2015.

U.S. Department of Education. Protecting Students With Disabilities Frequently Asked Questions About Section 504 and the Education of Children with Disabilities. Available at www2.ed.gov/about/ offices/list/ocr/504faq.html Accessed December 11, 2016.

Weisman H, Qureshi IA, Leckman JF, Scahill L, Bloch MH: Systematic review: Pharmacological treatment of tic disordersefficacy of antipsychotic and alpha-2 adrenergic agonist agents. Neurosci Biobehav Rev 37:1162-1171, 2013.

Wile DJ, Pringsheim TM: Behavior therapy for tourette syndrome: A systematic review and meta-analysis. Curr Treat Options Neurol 15:385-395, 2013.

Address correspondence to:

Margo Thienemann, MD

Stanford University

700 Welch Road, Suite 301

Palo Alto, CA 94305

E-mail: mthiene@stanford.edu 